\title{
Algorithms for Routing Optimization in Multipoint to Multipoint 4PL System
}

\author{
Jia Li, ${ }^{1}$ Yanqiu Liu, ${ }^{1}$ Ying Zhang, ${ }^{2}$ and Shida $\mathrm{Xu}{ }^{1}$ \\ ${ }^{1}$ School of Management, Shenyang University of Technology, Shenyang, Liaoning 110870, China \\ ${ }^{2}$ School of Science, Shenyang University of Technology, Shenyang, Liaoning 110870, China \\ Correspondence should be addressed to Jia Li; simsforever@sina.com
}

Received 4 December 2014; Accepted 29 December 2014

Academic Editor: Qingang Xiong

Copyright (c) 2015 Jia Li et al. This is an open access article distributed under the Creative Commons Attribution License, which permits unrestricted use, distribution, and reproduction in any medium, provided the original work is properly cited.

Fourth party logistics (4PL) has been receiving great attention and studied by researchers. This paper studies multipoint to multipoint 4PL system routing optimization with reliability constraints. Objective factors which cause disturbances of transportation time are taken into consideration. The mathematical model of the multipoint to multipoint multitask routing optimization in 4PL system with reliability constraints is set up. To solve the model, a Messy Genetic Algorithm (Messy GA) with double arrays encoding method is designed. The experimental result is compared with the Enumeration Algorithm (EA), and it shows that this algorithm can find the satisfactory solution and is a powerful optimization method for solving the problem.

\section{Introduction}

With the rapid development of global economy, trade between enterprises grows remarkably. Hence the ability to integrate supply chain for logistics companies has to increase. They are eager to a logistics company that owns strong comprehensive strength to provide scheme for the whole supply chain and be able to meet multiproduct distribution services from different production places to different demand places. Then, the fourth party logistics (4PL) appeared [1]. The operation essence and the core superiority of $4 \mathrm{PL}$ lie in its ability to integrate the supply chain resources. Then through integrating the most high-quality supply chain resources, the flexibility of the supply chain will get to a full extent, the customers will acquire the personalized and diversified integration solutions, and the fast, high-quality, and low-cost logistics services will be provided [2]. Meanwhile, with the increasing complexity of logistics network, its reliability has increasingly become one focus of business concern. However, the current planning and design of logistics network take little customers' demand of system reliability.

Many researchers have studied the $4 \mathrm{PL}$ and it has received increasing attention [3-8]. The routing problem of $4 \mathrm{PL}$ is management and optimization process from suppliers to demanders, which is directly related to the operational efficiency of 4PL. Most of these researches draw on graph-based network methods $[9,10]$. In [11], a target system of transporters is put forward and a hierarchy model for $4 \mathrm{PL}$ is established. A conceptual model that is based on research of $4 \mathrm{PL}$ implementation is presented in [12]. In [13], the authors consider several characteristics and issues related to horizontal collaboration implementation through an exploratory investigation of retailers, suppliers, and logistics service providers in the UK grocery retailing sector, focusing specifically on the role of fourth party logistics service providers. In [14], a tabu search algorithm for model of integration of $4 \mathrm{PL}$ is established.

Multipoint to multipoint and multitask problem in $4 \mathrm{PL}$ system with route selection and 3PL supplier's selection integrated is considered in this paper. It is a complex system. We take objective factors that cause disturbances of transportation time into consideration, and the mathematical model is set up. Our goal is minimizing the total cost in this multitask problem, and all tasks must satisfy reliability constraints that customer required. Because the multipoint to multipoint 4PL system routing problem is NP-hard two algorithms are designed: Messy Genetic Algorithm (Messy GA) and Enumeration Algorithm (EA). The algorithm is coded in Matlab 


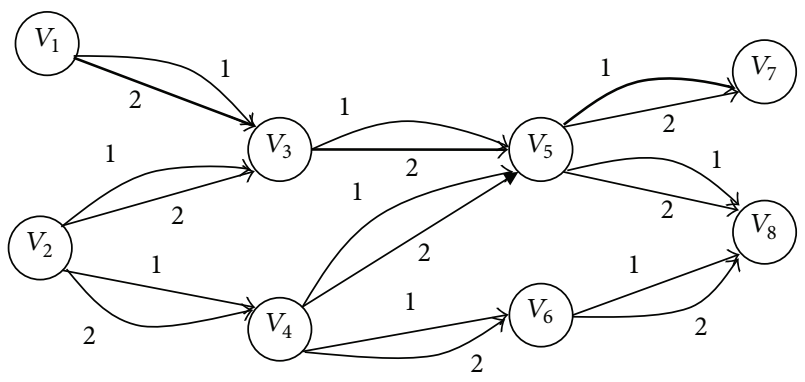

FIGURE 1: Directed graph with 8 nodes.

7.0 and run on a Core $22.83 \mathrm{GHz}$ computer. The comparisons in simulation testify the algorithms' effectiveness.

\section{Description of Problem and Mathematical Model}

2.1. Description of Problem. We use a straightforward way to describe the 4PL logistics system, which is multigraph. It can easily tell us all information needed clearly, as Figure 1 shows. The multigraph can be defined by $G(V, A) . V$ denotes the set of supply, transshipment, and demand nodes. A denotes the set of the arcs, namely, 3PL providers for transportation between two cities. $V_{1}$ and $V_{2}$ are supply nodes, $V_{7}$ and $V_{8}$ are demand nodes, and other nodes are transshipment nodes. There may many ways and 3PL providers between two locations, so multiple arcs exist between two nodes in multigraph. Each arc has the properties of cost, time, and capacity. In this paper, the objective of the problem is to find routes (i.e., Origin-Destination pair) with minimum cost as well as subject to constraints on customer's requirement for reliability and capacity.

2.2. The Reliability of $4 P L$ System. The route of $4 \mathrm{PL}$ system is composed of cities and 3PL providers. It forms a parallel system. According to reliability theory, the reliability of $4 \mathrm{PL}$ system can be calculated by continued multiplication of the reliability of 3PL. Here, each 3PL is seen as a logistics unit. In this paper, the transportation time of the 3PL providers is uncertain and has normal distribution. Hence, the time reliability of logistics unit can be defined as the probability of the goods delivered in a timely manner. It can be calculated by the following formula:

$$
R(t)=P(T \leq t)=\frac{1}{\sqrt{2 \pi} \sigma} \int_{0}^{t} e^{-(t-\mu)^{2} / 2 \sigma^{2}} d t \quad t>0
$$

$R(t)$ is the time reliability of logistics unit. $T$ is the transportation time of logistics unit. We assume $T \sim N\left(\mu, \sigma^{2}\right) . t$ is the time limit of the customer's requirements.

Another important influence factor of reliability is transportation cost. It can be calculated by the following formula:

$$
R(c)=e^{-1 / c}
$$

Here the reliability of logistics unit function is given as follows:

$$
R(t, c)=\alpha_{1} R(t)+\alpha_{2} R(c) \quad \alpha_{1}+\alpha_{2}=1
$$

$\alpha_{1}, \alpha_{2}$ are the weighting coefficients of the transportation time and the transportation cost separately.

The reliability of $4 \mathrm{PL}$ system can be calculated by the following formula:

$$
R_{S}=\prod_{i=1}^{n} R_{i}(t, c)
$$

$R_{S}$ is the reliability of $4 \mathrm{PL}$ system. $n$ is the number of the logistics unit in the $4 \mathrm{PL}$ system.

\subsection{Assumption of Model}

(i) There is not only one task between the supply and demand nodes.

(ii) Each path on the 4PL system cannot be reused.

(iii) The transportation time of $3 \mathrm{PL}$ providers is uncertain and has normal distribution. It is independent from each other.

2.4. Establishment of Model. Variables and parameters are defined as follows:

$V$ : set of nodes including suppliers, transit facilities, and demanders, $V=O \cup W \cup D$;

$O$ : sets of suppliers;

$W$ : set of transit nodes;

$D$ : set of demanders;

$q_{i j}$ : the number of 3PL provider between nodes $i$ and $j$

$a_{i j k}$ : the $k$ th 3PL provider between nodes $i$ and $j(i, j=$ $1,2, \ldots, n)\left(k=1,2, \ldots, q_{i j}\right)$ :

$X_{i j k}^{m}= \begin{cases}1 \quad & \begin{array}{l}\text { if the } k \text { th arc between nodes } i \text { and } j \\ \text { is selected for task } m \\ 0 \quad \text { otherwise, }\end{array}\end{cases}$

$Y_{i}^{m}= \begin{cases}1 & \text { if node } i \text { is selected for task } m \\ 0 & \text { otherwise; }\end{cases}$

$C_{i j k}$ : the unit transportation cost of the $k$ th $3 \mathrm{PL}$ provider between nodes $i$ and $j$;

$C_{i}^{\prime}$ : the unit cost of node $i$;

$f^{o d m}$ : the total number of goods transported from the oth supply node to $d$ th demand node for task $m$;

$P_{i j k}$ : the transportation capacity of the $k$ th $3 \mathrm{PL}$ provider between nodes $i$ and $j$;

$Q_{i}$ : processing capacity of node $i$; 
$T_{i j k}$ : time of 3PL provider represented by $a_{i j k}, T_{i j k} \sim$ $N\left(\mu_{i j k}, \sigma_{i j k}^{2}\right)$;

$t_{i j}$ : time limit of nodes $i$ to $j$;

$R_{i j k}$ : reliability of $a_{i j k} ; R_{i j k}=\alpha_{1}(1 /$ $\left.\sqrt{2 \pi} \sigma_{i j k}\right) \int_{0}^{t_{i j}} e^{-\left(t-\mu_{i j k}\right)^{2} / 2 \sigma_{i j k}^{2}} d t+\alpha_{2} e^{-1 / C_{i j k}}, \alpha_{1}+\alpha_{2}=1$;

$R_{\mathrm{OD}}$ : reliability of $\mathrm{OD}$ pair; $R_{\mathrm{OD}}=$ $\prod_{i=1}^{n} \prod_{j=1}^{n} \prod_{k=1}^{q_{i j}}\left[\alpha_{1}\left(1 / \sqrt{2 \pi} \sigma_{i j k}\right) \int_{0}^{t_{i j}} e^{-\left(t-\mu_{i j k}\right)^{2} / 2 \sigma_{i j k}{ }^{2}} d t+\right.$ $\left.\alpha_{2} e^{-1 / C_{i j k}}\right] \cdot X_{i j k}$;

$R_{0}$ : desired reliability level.

The mathematical model for $4 \mathrm{PL}$ system routing optimization with reliability constraints can be described as follows:

$$
\begin{array}{ll}
\min [ & {\left[\sum _ { o = 1 } ^ { O } \sum _ { d = 1 } ^ { D } \sum _ { m = 1 } ^ { M } \left(\sum_{i=1}^{n} \sum_{j=1}^{n} \sum_{k=1}^{q_{i j}} C_{i j k} \cdot f^{o d m} \cdot X_{i j k}^{m}\right.\right.} \\
& \left.\left.+\sum_{i=1}^{n} C_{i}^{\prime} \cdot f^{o d m} \cdot Y_{i}^{m}\right)\right] \\
\text { s.t. } \quad & \prod_{i=1}^{n} \prod_{j=1}^{n} \prod_{k=1}^{q_{i j}}\left[\alpha_{1} \frac{1}{\sqrt{2 \pi} \sigma_{i j k}} \int_{0}^{t_{i j}} e^{-\left(t-\mu_{i j k}\right)^{2} / 2 \sigma_{i j k}{ }^{2}} d t\right. \\
& o=1,2, \ldots, O \quad d=1,2, \ldots, D \quad m=1,2, \ldots, M, \\
& \left.\sum_{o=1}^{O} \sum_{d=1}^{D} \sum_{m=1}^{M} f^{-1 / C_{i j k}}\right] \cdot X_{i j k} \geq R_{0} \\
& i, j \in\{1,2, \ldots, n\}, \quad k \in\left\{1,2, \ldots, q_{i j}\right\} \\
& \sum_{o=1}^{O} \sum_{d=1}^{D} \sum_{m=1}^{M} f^{o d m} \cdot Y_{i}^{m} \leq Q_{i j k}, i \in\{1,2, \ldots, n\}
\end{array}
$$

$$
\begin{aligned}
& \sum_{i=1}^{n} f^{o d m} \cdot X_{i j k}^{m} \\
& \quad-\sum_{i=1}^{n} f^{o d m} \cdot X_{j i k}^{m}= \begin{cases}-f^{o d m} & \text { if } j=1 \\
f^{o d m} & \text { if } i=n \\
0 & \text { otherwise }\end{cases} \\
& j \in\{1,2, \ldots, n\}, \quad k \in\left\{1,2, \ldots, q_{i j}\right\} \\
& o=1,2, \ldots, O \quad d=1,2, \ldots, D, \\
& m=1,2, \ldots, M,
\end{aligned}
$$

$$
\begin{aligned}
& \sum_{i=1}^{n} \sum_{k=1}^{q_{i j}} X_{i j k}^{m}=Y_{j}^{m} \quad j \in\{2,3, \ldots, n\} \\
& m=1,2, \ldots, M, \\
& \sum_{i=1}^{n} \sum_{k=1}^{q_{i j}} X_{i j k}^{m}=Y_{i}^{m} \quad i \in\{1,2, \ldots, n-1\} \\
& m=1,2, \ldots, M, \\
& X_{i j k}^{m}=0 \text { or } 1, \quad i, j \in\{1,2, \ldots, n\}, \\
& k \in\left\{1,2, \ldots, q_{i j}\right\}, \quad o=1,2, \ldots, O \\
& d=1,2, \ldots, D \quad m=1,2, \ldots, M, \\
& Y_{i}^{m}=0 \text { or } 1, \quad i \in\{1,2, \ldots, n\}, o=1,2, \ldots, O \\
& d=1,2, \ldots, D \quad m=1,2, \ldots, M,
\end{aligned}
$$

where (6) is the objective function containing the costs of all nodes and arcs on the route; constraint (7) indicates the reliability of $O D$ pair must meet customer's requirement; constraint (8) represents capacity of the selected 3PL provider must be not less than the transportation capacity $f^{o d m}$ required by customer; constraint (9) denotes capacity of nodes in a route must be not less than customer required capacity. Equation (10) means to keep a balance of the network flow. Equations (11) and (12) ensure that the selected nodes and arcs should be made up of routes from the source to the destination. Equations (13) and (14) represent that $X_{i j k}^{m}$ and $Y_{i}^{m}$ are $0-1$ decision variables, respectively.

\section{Algorithm Design}

The multipoint to multipoint $4 \mathrm{PL}$ system routing optimization is a typical NP-hard problem. According to the characteristics of the model, a Messy Genetic Algorithm (Messy GA) with double arrays encoding method is designed. In the following, we first describe the Messy GA in detail and then introduce an Enumeration Algorithm (EA), which is used to check the quality of the solutions obtained by the Messy GA.

3.1. The Messy GA. According to the characteristics of the model, a Messy GA with double arrays encoding method is designed. Messy GA differ from normal genetic algorithms in that they allow variable-length strings. The detailed description of the process can be written as below.

Step 1 (encoding). For the problem's characteristic, the double arrays coding method is adopted where optional nodes in graph are the elements of one array coded by natural number and 3PL providers make up the other array coded by integer number and the length of individuals is variant. The bold line in Figure 1 can be encoded as $\left[V_{1} V_{3} V_{5} V_{7} a_{2} a_{2} a_{1}\right]$.

Step 2 (initialization). Generate the initial population of PS chromosomes randomly. Population size is PS. 
Step 3 (fitness function). In this paper, reliability constraint is used as a penalty added to the objective function, and the $\omega$ is a penalty factor. The fitness function is represented by (15).

The values of $\omega$ range from 1 to 2 , depending on the actual situation:

$$
\begin{aligned}
& f(X, Y) \\
& =\sum_{o=1}^{O} \sum_{d=1}^{D} \sum_{m=1}^{M}\left(\sum_{i=1}^{n} \sum_{j=1}^{n} \sum_{k=1}^{q_{i j}} C_{i j k} \cdot f^{o d m} \cdot X_{i j k}^{m}\right. \\
& \left.+\sum_{i=1}^{n} C_{i}^{\prime} \cdot f^{o d m} \cdot Y_{i}^{m}\right) \\
& +\left\{\sum _ { o = 1 } ^ { O } \sum _ { d = 1 } ^ { D } \sum _ { m = 1 } ^ { M } \left(\sum_{i=1}^{n} \sum_{j=1}^{n} \sum_{k=1}^{q_{i j}} C_{i j k} \cdot f^{o d m} \cdot X_{i j k}^{m}\right.\right. \\
& \left..\left\{\lambda+R_{0}^{n}+\sum_{i=1}^{n} C_{i}^{\prime} \cdot f^{o d m} \cdot Y_{i}^{m}\right)\right\} \\
& +\prod_{i=1}^{n} \prod_{j=1}^{n} \prod_{k=1}^{q_{i j}}\left[\alpha_{1} \frac{1}{\sqrt{2 \pi} \sigma_{i j k}}\right]_{0}^{t_{i j}} e^{-\left(t-\mu_{i j k}\right)^{2} / 2 \sigma_{i j k}{ }^{2}} d t
\end{aligned}
$$

Step 4 (selection mechanism). The selection process is based on spinning the roulette wheel.

Step 5 (crossover operation). Perform crossover operations on pairs of selected chromosomes with a crossover probability $P_{c}$. After cut operator and splicing operator, chromosomes $A^{\prime \prime}$ and $B^{\prime \prime}$ may be invalid. Therefore, repairing operation is necessary. The crossover procedure is shown in Figure 2. We need to adjust the chromosome to ensure that it is a connective route. Detailed description of the process can be written as below.

Step 5.1. If there are same nodes on the route, delete one node and the adjacent arc. If not, go to Step 5.2.

Step 5.2. If the new one is still a route from the source to the destination, quit with success. If not, add all the nodes after the crossover point into the Set ${ }_{p}$. Define the node before the crossover point as the tracker node $N_{t}$. Define the genes before the crossover point as the effective route $L$.

Step 5.3. If the node $N_{t} \in \operatorname{Set}_{p}$, splice the route $L$ with the route after the node which is $N_{t} \in \operatorname{Set}_{p}$, and if the new chromosome is the legal route, quit with success. If not, select one node adjacent to the tracker node $N_{t}$ as the new tracker node $N_{t+1}$, and add the new arc to the effective route $L$.

Step 5.4. Determine if the trace node $N_{t+1}$ is the destination node $D$; if it is a valid route, $L$ becomes the new chromosome, so quit with success; if not, turn to Step 5.3.

Step 6 (mutation operation). For performing mutation operation in the feasible solution space, select a chromosome randomly, and change the arcs of the adjacent nodes in $P_{m}$ probability of mutation operation.

Step 7 (termination criterion). Check whether iteration times are over NG. If it is true, stop the process and output the optimum solution; else turn to Step 3.

3.2. The Enumeration Algorithm. To check the quality of the solutions obtained by the Messy GA, the Enumeration Algorithm (EA) is used. Because the solution space is very large, the EA is described as follows.

Step 1. Produce all the routes from the supply node $O$ and the demand node $D$ on the direct graphs that satisfy the requirements of capacity and reliability.

Step 2. Calculate the fitness function value of every route. The fitness function is designed according to the objective function and reliability constraint of the model. And it is similar to the fitness function of the Messy GA in (15).

Step 3. All the combinations of routes are checked and ordered according to the fitness function. The combination with the lowest fitness function value is the best solution.

\section{Numerical Analysis}

Assume that a $4 \mathrm{PL}$ company undertakes a transnational transportation. In this transportation, we need to transport 3 tons of food and 5 tons of coal from the supply nodes $V_{1}$ and $V_{2}$ to the demand nodes $V_{7}$ and $V_{8}$. The topological structure is shown in Figure 1.

The algorithm is coded in Matlab 7.0 and run on a Core $22.83 \mathrm{GHz}$ computer $[15,16]$. We analyze the performance of Messy GA and compare the result with that of Enumeration Algorithm (EA). The parameters affecting the performance include population size (PS), number of generation (NG), crossover probability $\left(P_{c}\right)$, and scaling factor of mutation $\left(P_{m}\right)$. Desired reliability level $\left(R_{0}\right)$ is 0.85 . The costs of the nodes and the arcs in the following are recorded by 1000 dollars. The capacity is recorded by ton, and the time of $3 \mathrm{PL}$ provider is recorded by hour. The parameters are set below:

$$
\begin{aligned}
& \omega=3, \quad \mathrm{PS}=30, \quad \mathrm{NG}=300, \quad P_{c}=0.5, \\
& P_{m}=0.15, \quad \alpha_{1}=\alpha_{2}=0.5 \text {. }
\end{aligned}
$$

The node and arc data are given in Tables 1 and 2, where " $V_{i}$ " and " $V_{j}$ " mean the start and end nodes, respectively.

The experimental results are shown in Table 3.

The 4PL company needs to transport food and coal from supply nodes to demand nodes with the reliability not less than 0.85 . From Table 3, it can also be seen that both Messy 

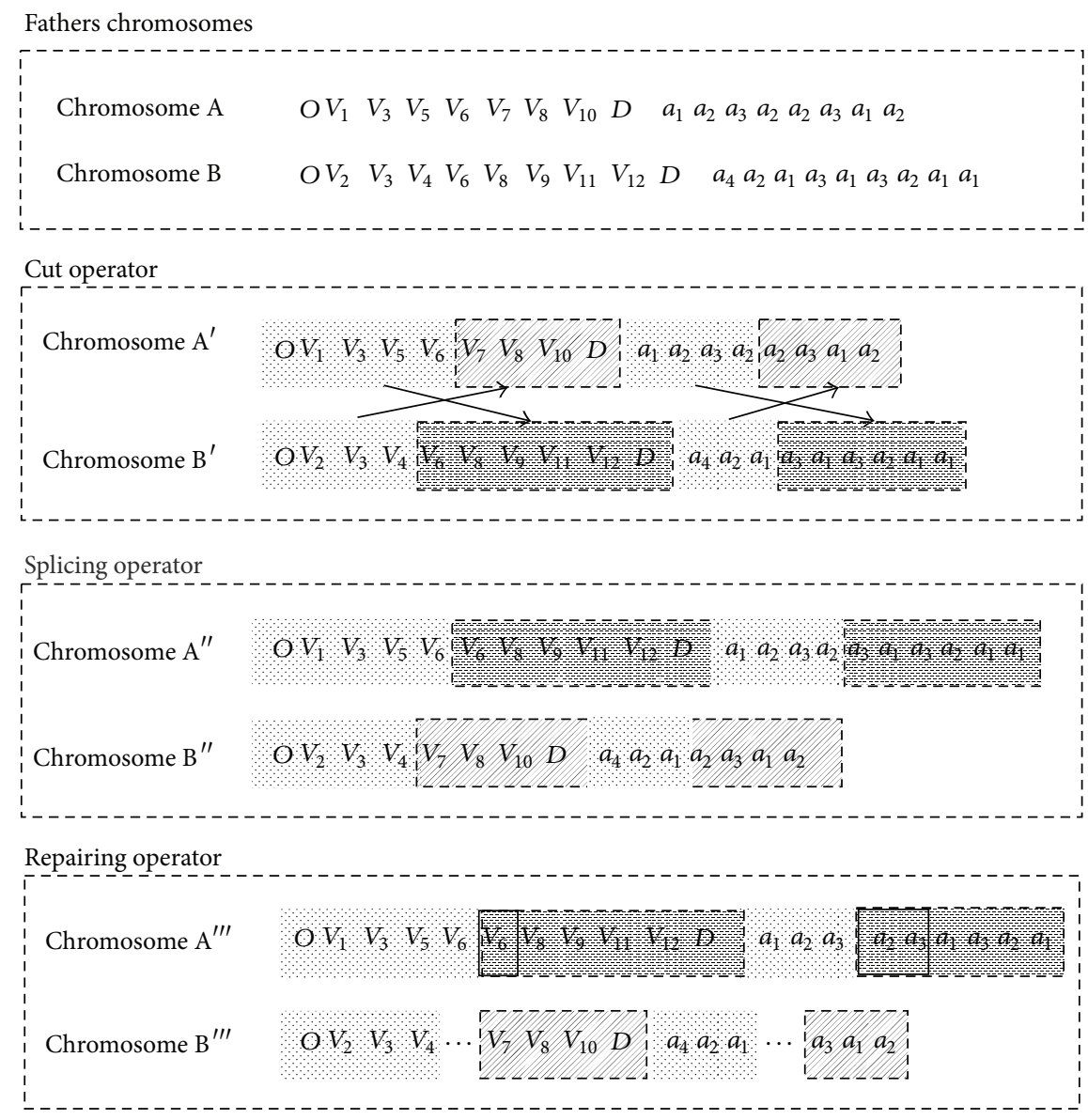

Children chromosomes

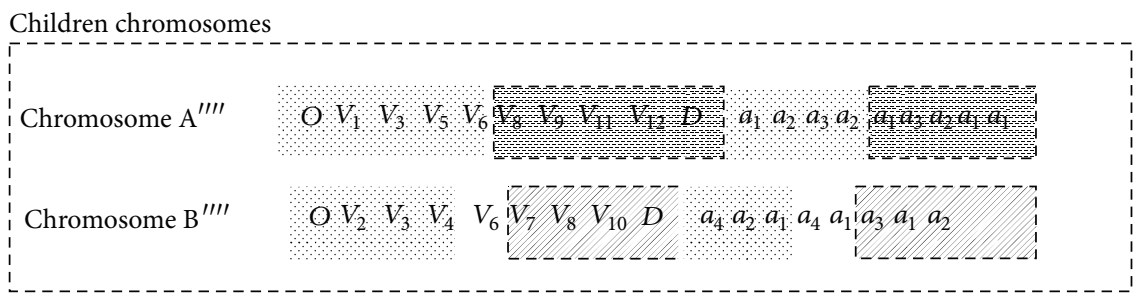

FIgURE 2: Crossover operation.

TABLE 1: The data of nodes for the 8-node problem.

\begin{tabular}{lcc}
\hline Node & Cost (1000 dollars) & Capacity (ton) \\
\hline$V_{1}$ & 5 & 9 \\
$V_{2}$ & 4 & 8 \\
$V_{3}$ & 3 & 10 \\
$V_{4}$ & 6 & 12 \\
$V_{5}$ & 4 & 9 \\
$V_{6}$ & 3 & 8 \\
$V_{7}$ & 2 & 10 \\
$V_{8}$ & 2 & 12 \\
\hline
\end{tabular}

GA and EA can obtain optimal result, but Messy GA can solve the problem quickly.

\section{Conclusions}

The routing optimization in $4 \mathrm{PL}$ system is one of the most important problems in supply chain optimization. This study set up the mathematical model of the multipoint to multipoint 4PL system routing optimization with reliability constraints based on the reliability theory. The Messy GA and the EA are proposed to solve the model. Numerical experiments are carried out to investigate the performance of the proposed Messy GA. The experiment results show that the Messy GA can obtain the same best result as the EA.

\section{Conflict of Interests}

The authors declare that there is no conflict of interests regarding the publication of this paper. 
TABLE 2: The data of arcs for the 8-node problem.

\begin{tabular}{|c|c|c|c|c|c|c|c|c|c|}
\hline$V_{i}$ & $V_{j}$ & $a_{i j k}$ & $C_{i j k}$ & $P_{i j k}$ & $T_{i j k} \sim N\left(\mu_{i j k}, \sigma_{i j k}^{2}\right)$ & $t_{i j}$ & $R(t)$ & $R(c)$ & $R_{i j k}$ \\
\hline \multirow{2}{*}{1} & \multirow{2}{*}{3} & 1 & 18 & 12 & $N(18,5)$ & 24 & 0.88493 & 0.945959 & 0.915445 \\
\hline & & 2 & 18 & 10 & $N(16,6)$ & 24 & 0.908789 & 0.945959 & 0.927374 \\
\hline \multirow{4}{*}{2} & \multirow{2}{*}{3} & 1 & 18 & 11 & $N(16,8)$ & 24 & 0.841345 & 0.945959 & 0.893652 \\
\hline & & 2 & 18 & 12 & $N(17,6)$ & 24 & 0.878327 & 0.945959 & 0.912143 \\
\hline & \multirow{2}{*}{4} & 1 & 17 & 10 & $N(18,4)$ & 24 & 0.933193 & 0.942873 & 0.938033 \\
\hline & & 2 & 20 & 12 & $N(16,7)$ & 24 & 0.873451 & 0.951229 & 0.91234 \\
\hline \multirow{2}{*}{3} & \multirow{2}{*}{5} & 1 & 18 & 9 & $N(20,5)$ & 24 & 0.788145 & 0.945959 & 0.867052 \\
\hline & & 2 & 17 & 10 & $N(20,6)$ & 36 & 0.99617 & 0.942873 & 0.969521 \\
\hline \multirow{4}{*}{4} & \multirow{2}{*}{5} & 1 & 18 & 15 & $N(25,7)$ & 36 & 0.941958 & 0.945959 & 0.943959 \\
\hline & & 2 & 12 & 12 & $N(12,4)$ & 18 & 0.933193 & 0.920044 & 0.926619 \\
\hline & \multirow{2}{*}{6} & 1 & 13 & 14 & $N(11,4)$ & 18 & 0.959941 & 0.925961 & 0.942951 \\
\hline & & 2 & 16 & 13 & $N(13,2)$ & 18 & 0.99379 & 0.939413 & 0.966602 \\
\hline \multirow{4}{*}{5} & \multirow{2}{*}{7} & 1 & 17 & 11 & $N(15,3)$ & 18 & 0.841345 & 0.942873 & 0.892109 \\
\hline & & 2 & 10 & 14 & $N(14,5)$ & 20 & 0.88493 & 0.904837 & 0.894884 \\
\hline & \multirow{2}{*}{8} & 1 & 12 & 16 & $N(13,3)$ & 20 & 0.990185 & 0.920044 & 0.955115 \\
\hline & & 2 & 12 & 17 & $N(13,4)$ & 20 & 0.959941 & 0.920044 & 0.939993 \\
\hline \multirow{2}{*}{6} & \multirow{2}{*}{8} & 1 & 15 & 10 & $N(16,5)$ & 24 & 0.945201 & 0.935507 & 0.940354 \\
\hline & & 2 & 10 & 9 & $N(13,4)$ & 24 & 0.99702 & 0.904837 & 0.950929 \\
\hline
\end{tabular}

TABLE 3: Comparison of two algorithms.

\begin{tabular}{lclcc}
\hline Algorithm & Total cost (1000 dollars) & Optimal route & Reliability of the route & Run time (second) \\
\hline \multirow{2}{*}{ Messy GA } & \multirow{2}{*}{357} & $V_{1} V_{3} V_{5} V_{7} a_{2} a_{2} a_{1}$ (FOOD) & 0.859 (FOOD) & $<1$ \\
& & $V_{2} V_{4} V_{6} V_{8} a_{1} a_{2} a_{2}$ (COAL) & 0.862 (COAL) & \\
\hline \multirow{2}{*}{ EA } & \multirow{2}{*}{357} & $V_{1} V_{3} V_{5} V_{7} a_{2} a_{2} a_{1}$ (FOOD) & 0.859 (FOOD) & \\
& & $V_{2} V_{4} V_{6} V_{8} a_{1} a_{2} a_{2}$ (COAL) & $0.862(\mathrm{COAL})$ & \\
\hline
\end{tabular}

\section{Acknowledgments}

This paper is supported by the Science and Technology Support Program of Liaoning Province (no. 2013216015) and the Science and Technology Support Program of Shenyang (no. F13-051-2-00 and no. F14-231-1-24).

\section{References}

[1] H. Min, R. Liang, C. Ming, and C. Yan, "Modeling and optimizing of 4PL routing optimization problem based on goal programming," in Proceedings of the 25th Chinese Control and Decision Conference (CCDC '13), pp. 2348-2352, IEEE, May 2013.

[2] J. M. Yao, "Decision optimization analysis on supply chain resource integration in fourth party logistics," Journal of Manufacturing Systems, vol. 29, no. 4, pp. 121-129, 2010.

[3] W. Li, M. Y. Wu, and Q. Mei, "The research of supply chain based on fourth party logistics optimization," in Proceedings of the International Conference on Building Materials and Structural Engineering, pp. 393-397, 2012.

[4] J. G. Shao and Q. Chen, "The advantages and development strategies of the fourth party logistics in China," in Proceedings of the 2nd International Conference on Engineering and Business Management, pp. 923-928, 2011.

[5] J. Q. Chen, W. H. Liu, and X. Li, "The directed graph model with multi dimensions in the fourth party logistics and its algorithm,"
Industrial Engineering and Management, vol. 8, no. 3, pp. 45-49, 2003.

[6] L. Chen, L. Yang, and B. Sun, "Operation mode selection of fourth-party logistics in China," Advances in Intelligent and Soft Computing, vol. 143, pp. 499-506, 2012.

[7] C. Zhang and J. E. Ramirez-Marquez, "Approximation of minimal cut sets for a flow network via evolutionary optimization and data mining techniques," International Journal of Performability Engineering, vol. 7, no. 1, pp. 21-31, 2011.

[8] Y.-K. Lin, "Multiple commodity flow problem with unreliable nodes subject to budget constraints," International Journal of Performability Engineering, vol. 4, no. 1, pp. 31-42, 2008.

[9] X. W. Wang, L. Guo, C. Q. Yu et al., "A new heuristic protection algorithm based on survivable integrated auxiliary graph in waveband switching optical networks," Computer Communications, vol. 32, no. 5, pp. 1000-1005, 2009.

[10] L. Guo, X. W. Wang, J. N. Cao, W. G. Hou, J. J. Wu, and Y. Li, "Local and global hamiltonian cycle protection algorithm based on abstracted virtual topology in fault-tolerant multi-domain optical networks," IEEE Transactions on Communications, vol. 58, no. 3, pp. 851-859, 2010.

[11] H. Zhang, J. Q. Chen, W. H. Liu et al., "Appraisement of transporters in fourth party logistics," Industrial Engineering Journal, vol. 7, no. 3, pp. 36-39, 2004.

[12] A. Win, "The value a $4 \mathrm{PL}$ provider can contribute to an organization," International Journal of Physical Distribution \& Logistics Management, vol. 38, no. 9, pp. 674-684, 2008. 
[13] M. Hingley, A. Lindgreen, D. B. Grant, and C. Kane, "Using fourth-party logistics management to improve horizontal collaboration among grocery retailers," Supply Chain Management, vol. 16, no. 5, pp. 316-327, 2011.

[14] Y. Wang, H. Zhao, and Y. Li, "Tabu search algorithm for optimization model of integration of job of 4th party logistics," Journal of Systems Engineering, vol. 21, no. 2, pp. 143-149, 2006.

[15] Q. Xiong, S. Aramideh, A. Passalacqua, and S. C. Kong, "BIOTC: an open-source CFD code for simulating biomass fast pyrolysis," Computer Physics Communications, vol. 185, no. 6, pp. 1739-1746, 2014.

[16] Q. Xiong, S. C. Kong, and A. Passalacqua, "Development of a generalized numerical framework for simulating biomass fast pyrolysis in fluidized-bed reactors," Chemical Engineering Science, vol. 99, pp. 305-313, 2013. 


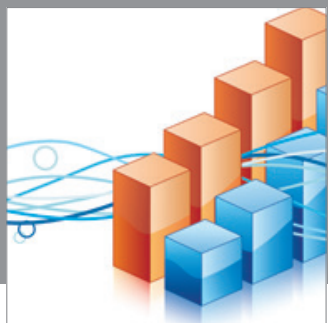

Advances in

Operations Research

mansans

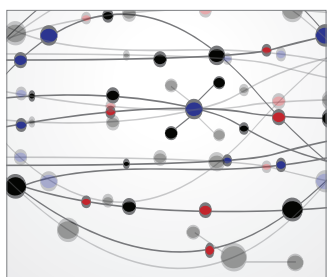

The Scientific World Journal
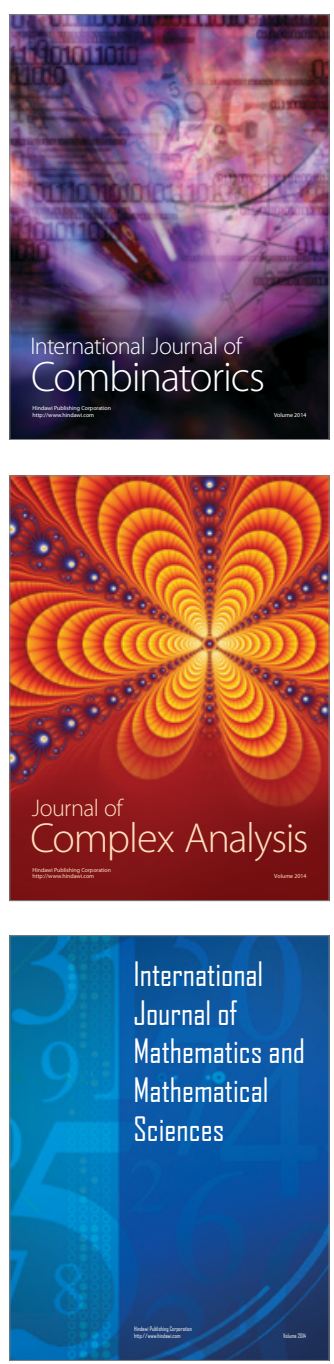
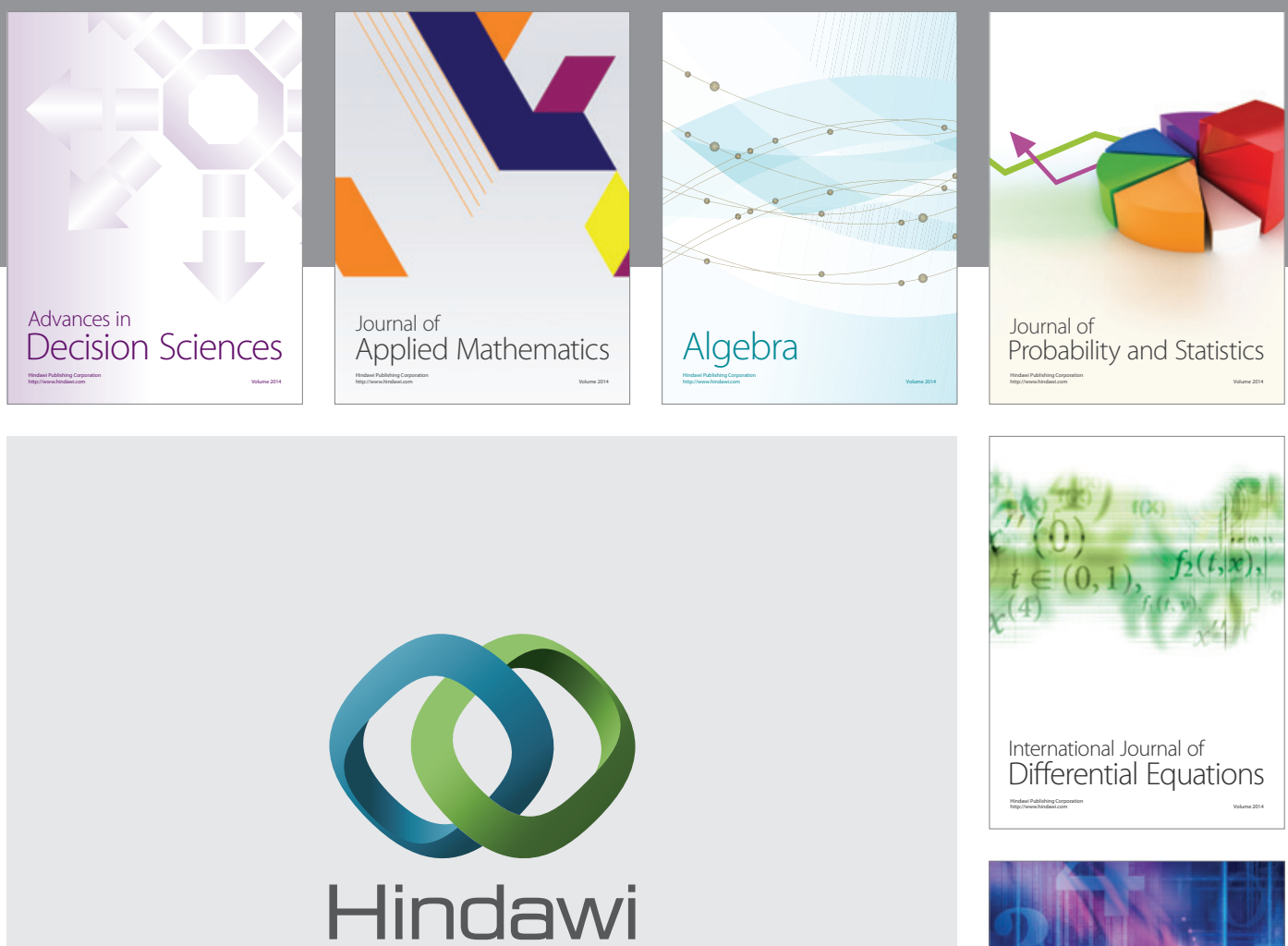

Submit your manuscripts at http://www.hindawi.com
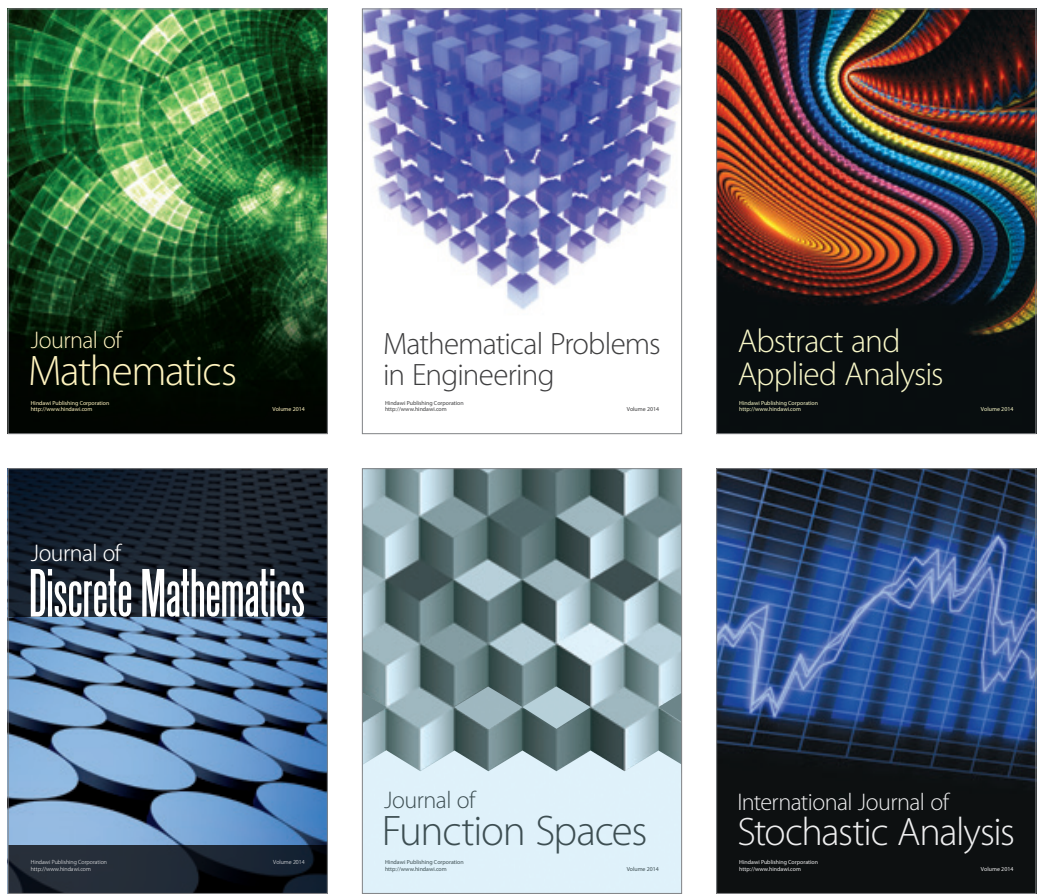

Journal of

Function Spaces

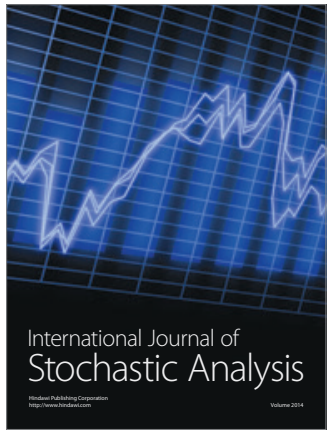

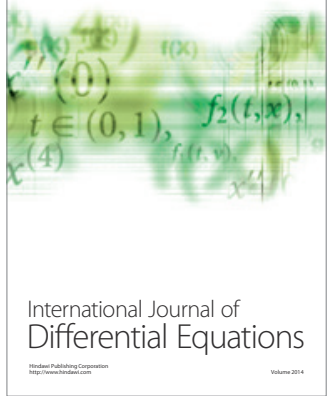
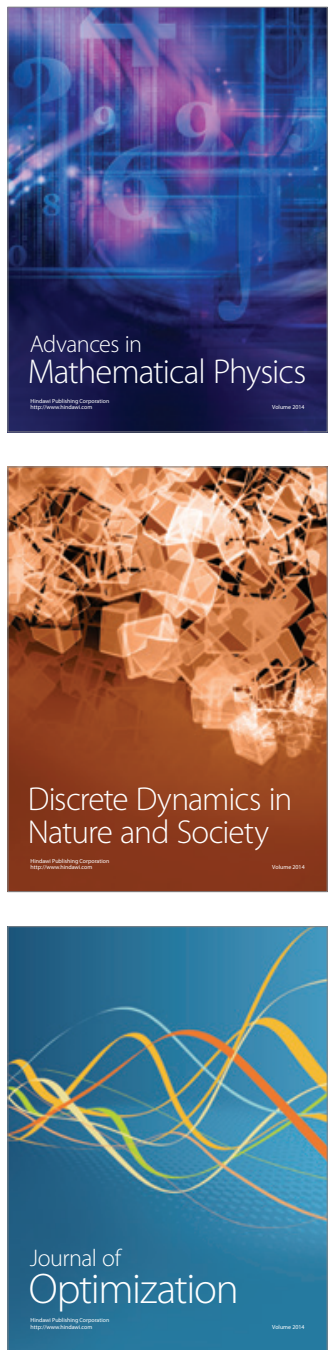